improved R0 resection rates at primary cytoreductive surgery. This study hasdescribed select the patients that would achieve the most benefit from primary surgery.

Laparoscopic scoring allowed for a more personalized approach to the management of patients with advanced-stage ovarian cancer at our institution. It resulted in an objective triage of patients to primary cytoreduction or NACT, and improved R0 resection rates at primary cytoreductive surgery. The present study has designed to assess the patients that would experience the maximum benefits from primary surgery.

\section{HOW TO DO RETROPERITONEAL EN BLOC POSTERIOR PELVIC EXENTERATION IN ADVANCED OVARIAN CANCER: HUDSON-DELLEPIANE PROCEDURE IN 10 STEPS}

${ }^{1} \mathrm{~N}$ Bizzarri ${ }^{*},{ }^{1} \mathrm{~B}$ Marinucci, ${ }^{1,2} \mathrm{~S}$ Restaino, ${ }^{1} \mathrm{G}$ Scambia, ${ }^{1} \mathrm{~A}$ Fagotti, ${ }^{1} \mathrm{~F}$ Fanfani. ${ }^{1}$ Fondazione Policlinico Universitario A. Gemelli, IRCCS, UOC Ginecologia Oncologica, Dipartimento per la salute della Donna e del Bambino e della Salute Pubblica, Rome, Italy; ${ }^{2}$ Academic Hospital of Udine, Obstetrics and Gynecology Department, Udine, Italy

\subsection{6/ijgc-2021-ESGO.480}

Introduction/Background* The aim of cytoreduction in advanced ovarian cancer is complete removal of gross disease. Retroperitoneal approach is crucial in radical surgery for advanced ovarian cancer. Hudson-Delle Piane is a radical procedure which allows en-bloc removal of uterus, ovaries, pouch of Douglas peritoneum, recto-sigmoid with a retrograde approach in cases where recto-simoid sparing is not an option.

Methodology We present an educational video on how to perform retroperitoneal en bloc posterior pelvic exenteration in advanced ovarian cancer with a step-by-step procedure. The patient was a 52-year-old woman, with body mass index of 21.1 who presented with abdominal pain and distension; the CT-scan showed peritoneal carcinomatosis. The different phases of the Fagotti's score performed at diagnostic laparoscopy to triage for the operability, are demonstrated. The Vizzielli's score was used to determine the risk of post-operative complications in case of primary debulking surgery is also presented.

Result(s)* Immediately after the diagnostic laparoscopy a conversion to laparotomy with a cytoreductive surgery was performed. The Hudson-Delle Piane technique is demonstrated in 10 steps. The surgery lasted 360 minutes and the estimated blood loss was $400 \mathrm{ml}$. No peri-operative complication was recorded. The histology revealed a FIGO stage IIIC high-grade serous ovarian cancer.

Conclusion* The present video demonstrates that retroperitoneal approach to advanced ovarian cancer with high volume pelvic disease, allows en bloc tumor debulking with safe handling of important structures.

\section{THE ROLE OF CYTOREDUCTIVE MULTIVISCERAL SURGERY IN OVARIAN GROWING TERATOMA SYNDROME - CASE SERIES}

${ }^{1} \mathrm{E}$ Lukacs*, 'Z Novák, ${ }^{2} \mathrm{~K}$ Bíró, ${ }^{2} \mathrm{~L}$ Géczi, ${ }^{2} \mathrm{~F}$ Gyergyay, ${ }^{1} \mathrm{~A}$ Bagaméri. ${ }^{1}$ National Institute of Oncology, Gynecology, Budapest, Hungary; ${ }^{2}$ National Institute of Oncology, Department of Genitourinary, Medical Oncology and Clinical Pharmacology (Chemotherapy "C"), Budapest, Hungary

10.1136/ijgc-2021-ESG0.481
Introduction/Background* Immature teratomas are rare germ cell ovarian tumours occuring in about $1 \%$ of ovarian cancers. Growing Teratoma Syndrome (GTS) is a rare clinical situation where seemingly successful chemotherapy is followed by a rapid tumor progression, while tumour markers normalize. Despite radiological progression, the phenomenon is characterised by the maturation of the teratoma, and in most cases there is hardly any immature component left. In these rare cases radical surgical treatment is usually effective and can lead to complete recovery.

Methodology case report

Result(s)* A joint working group of the Department of Gynecology and Department of Genitourinary, Medical Oncology and Clinical Pharmacology of the National Institute of Oncology, Hungary diagnosed three immature ovarian teratoma cases with extended pelvic/abdominal tumor mass which showed progressive growth during and following chemotherapy. In all three cases, we had to perform multivisceral cytoreductive surgery with peritonectomy leading to complete cytoreduction. All patients are currently tumor-free. Postoperative histological findings showed $80-100 \%$ maturation, one of them operated 19 years after the primary treatment.

Conclusion* By presenting these 3 patients, our goal is to raise awareness of the possibility of GTS and to emphasize the importance of adequate treatment.

\section{STUDY OF THE LYMPHATIC MAP AND DETECTION OF THE SENTINEL LYMPH NODE IN OVARIC MASSES WITH SUSPECTED MALIGNANCY}

N Agustí ${ }^{*}$ P Paredes, S Vidal-Sicart, AG Glickman, P Fusté, N Carreras, J Pahisa, M Del Pino, A Fristch, A Torne, B Diaz-Feijoo. Hospital Clínic de Barcelona, Barcelona, Spain

\subsection{6/ijgc-2021-ESGO.482}

Introduction/Background* Systematic pelvic and paraortic lymphadenectomy is part of the initial epithelial ovarian cancer (EOC) staging surgery but this procedure is associated with potential severe morbidity. Moreover, there is no evidence suggesting a possible therapeutic value. The detection of the sentinel lymph node (SLN) in the early stage of EOC is in the experimental phase and there is little literature about it. This study evaluates the lymphatic mapping with radiotracer and indocyanine green (ICG)) and the detection of ovarian SLN.

Methodology Prospective cohort study in ovarian masses suspected of malignancy or re-staging surgery after confirmed malignancy. Albumin [99mTc] Tc-nanocolloid was injected into the utero-ovarian and infundibulo-pelvic ligaments. After 15 minutes, intraoperative images were acquired with a portable gammacamera. Subsequently, the adnexectomy and intraoperative pathological analysis were performed. In the cases in which malignancy was confirmed, ICG was injected proximally to the sectioned area of the ligaments and the lymphatic chains were traced with the fluorescence camera prior to the pelvic and paraortic lymphadenectomy. Ultrastaging of the SLN was done. The follow-up for possible adverse events lasted 30 days after surgery.

Result(s)* 20 patients were included between September-2020 and May-2021. Ovarian carcinoma was confirmed in 8 (40\%) cases. In all cases gamma probe was used, in $60 \%$ the gamma camera and $40 \%$ the fluorescence camera. Some SLN was 


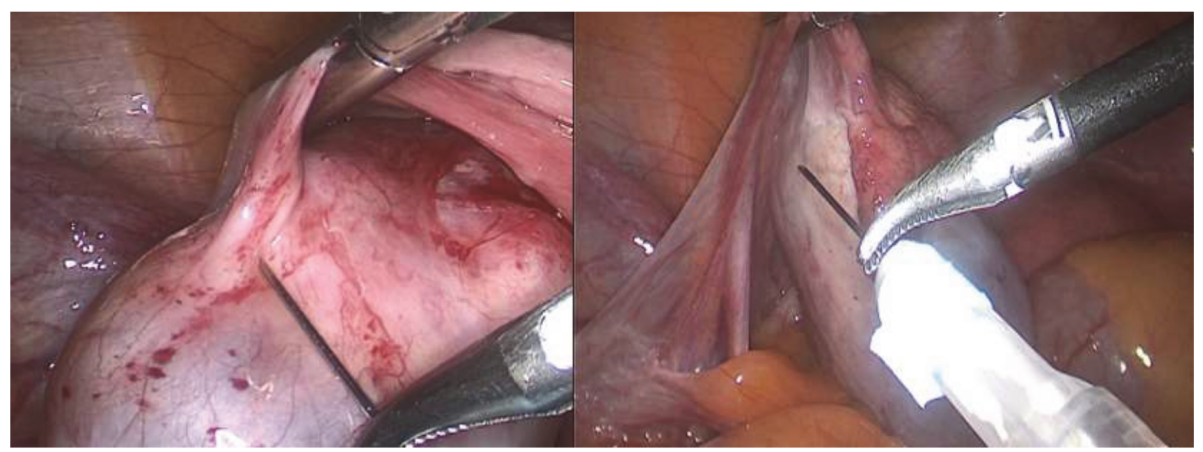

Abstract 834 Figure 1 Albumin [99mTc] Tc-nanocolloid injection into the utero-ovarian and infundibulo-pelvic ligaments
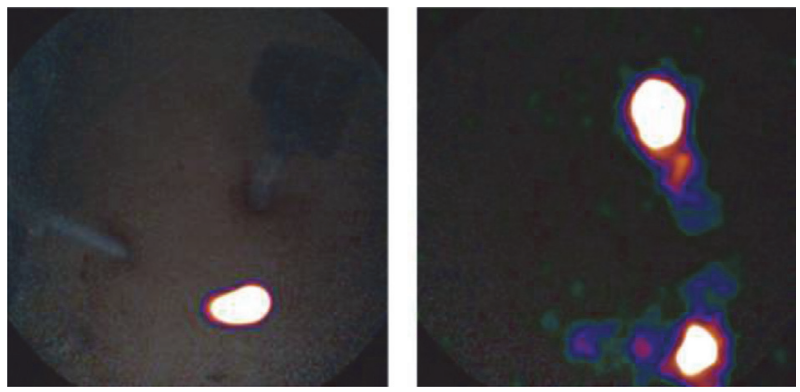

Abstract 834 Figure 2 Images acquired with the portable gamma camera at 15 minutes and at 90 minutes postinjection. Paraortic drainage is observed.

detected in 18/20 cases (90\%) with exclusive paraortic drainage in $5 / 18(28 \%)$, pelvic in $1 / 18(6 \%)$ and in both territories in $12 / 18(66 \%)$. Overall para-aortic drainage was observed in $17 / 18$ (94\%) patients (35\% supramesenteric, 30\% at the level of the inferior mesenteric artery, and $35 \%$ inframesenteric). $100 \%$ of aortic SLNs were detected with a gamma probe after being visualized with the gamma camera. In the 8 patients who underwent lymphadenectomy, 1 case had positive nodes diagnosed by ultrastaging and the rest of the lymph nodes were negative. No complications related to the technique were observed.

Conclusion* The SLN technique is feasible and safe. The intraoperative gamma camera shows the lymphatic map and is especially useful in the paraortic region.

\section{FEASIBILITY OF DIAPHRAGMATIC SURGERY FOR ADVANCED OVARIAN CANCER: A SINGLE INSTITUTION ANALYSIS}

G Candotti ${ }^{*}$, F Sgalambro, A Bergamini, F Cantatore, R Cioffi, P De Marzi, ML Fais, F Galli, E Rabaiotti, G Sabetta, C Saponaro, L Bocciolone. San Raffaele Hospital, Obstetrics and Gynecology, Milano, Italy

\subsection{6/ijgc-2021-ESGO.483}

Introduction/Background* Standard treatment for advanced ovarian cancer patients should be primary cytoreduction following platinum-based chemotherapy. The aim of surgical effort should be the complete removal of all macroscopic disease. Surgery of upper abdomen is often required to obtain optimal cytoreduction. Our objective was to evaluate perioperative features, postoperative complications of patients who underwent diaphragmatic stripping or diaphragmatic resection for advanced ovarian cancer.

Methodology From June 2018 to April 2021, 138 patients underwent cytoreductive surgery for advanced ovarian cancer at San Raffaele Hospital. Forty-one cases were selected, among them 29 underwent diaphragmatic stripping and 12 underwent diaphragmatic full-thickness resection during primary cytoreduction surgery (PCS) or interval debulking surgery (IDS). All surgical procedures were performed by use of bipolar scissors and blunted resection. Data collected included patients' age, all perioperative details and pathological findings, International Federation of Gynecology and Obstetrics stage.

Result(s)* Median age was 60 (range 32-79) years. Median hospital stays were 7 (range 4-26) days. Thirty-five patients $(85 \%)$ obtained optimal cytoreduction with absence of macroscopic disease. Mean loss of blood was $391 \mathrm{ml}( \pm 172)$. Thirtyfour (83\%) patients underwent PDS and 7 (17\%) underwent IDS. Postoperative pleural effusion rate was $14,6 \%$ without any differences between stripping or diaphragmatic resection $(p=0,423)$. One case of pneumothorax was reported in the stripping group.

Conclusion* Diaphragmatic surgery at the time of primary cytoreductive surgery or IDS for advanced ovarian cancer may contribute to the achievement of complete cytoreduction with low perioperative complication rate. Risk of postoperative pleural effusion could be limited by utilization of bipolar scissors and blunted resection to perform surgical procedures.

\section{CANCER-INDUCED ACCELERATED IMMUNOAGING IN WOMEN SUFFERING FROM OVARIAN CANCER}

S Marcoux*, T Kientega, GB Cardin, MF Raynault, AM Mes-Masson, D Provencher, F Rodier. Université de Montréal Faculty of Medicine, Médecine, Montreal, Canada

\subsection{6/ijgc-2021-ESG0.484}

Introduction/Background* Advanced age is a poor prognostic risk factor for most epithelial ovarian cancers. Individuals suffering from socioeconomic deprivation have a lower life expectation and a higher risk for most chronic diseases, including cancer. There is also mounting evidence that undergoing cancer treatments is associated with accelerated cellular aging. Hypothesis: extensive accelerated aging caused by 1) socioeconomic status, 2) cancer itself and 3) cancer therapy is associated with worse prognosis in women suffering from primary ovarian cancer.

Methodology We retrospectively analyzed the immunological age of 488 women diagnosed and treated for a primary 